\title{
Relationship Between the Coronary Artery Calcium (CAC) Score and the Angle Between the Aortic Valve and the Left Ventricular Inflow Long Axis (AV-LV) as Cardiovascular Risk Factors
}

\author{
Taraneh Faghihi (iD ${ }^{1}$, Azadeh Ehsani (iD ${ }^{1,}$, , Fatemeh Shojaeian (id ${ }^{2}$ and Seyed Amir Ahmad Safavi Naini \\ (iD) 2 \\ ${ }^{1}$ Radiology Department, Shahid Modarres Hospital, Shahid Beheshti University of Medical Science, Tehran, Iran \\ ${ }^{2}$ Imam Hossein Hospital Clinical Research Development Unit, Imam Hossein Hospital, Shahid Beheshti University of Medical Science, Tehran, Iran \\ "Corresponding author: Radiology Department, Shahid Modarres Hospital, Sa'adat Abaad Avenue, 1998734383, Tehran, Iran. Tel: +98-9126768409, Email: \\ ehsani.azade@gmail.com
}

Received 2020 October 19; Revised 2021 June 30; Accepted 2021 July 07.

\begin{abstract}
Background: Cardiovascular disease (CVD) is one of the leading causes of mortality in both developed and developing countries. Therefore, identification of CVD risk factors is one of the most important steps in preventing this disease. Evidence suggests that imaging of the cardiac anatomy can be helpful for risk assessment.

Objectives: This study aimed to assess two cardiac anatomy features, namely, the angle between the aortic valve and the left ventricular inflow long axis (AV-LV angle) and the coronary artery calcium (CAC) score.

Patients and Methods: This cross-sectional study was conducted on 74 patients with indications for computed tomography angiography (CTA) according to the American Heart Association (AHA) guidelines from July 2019 to January 2020 in Iran. The Agatston method was applied to measure the CAC score. Besides, to determine the AV-LV angle, the angle between a line tangent to the aortic valve leaflets and a line through the center of the aorta toward the left ventricular apex was measured. The patients' baseline characteristics were also documented by conducting interviews.

Results: Of 74 patients, $56 \%$ were male. The mean age of the patients was $54.41 \pm 12.31$ years, and their mean body mass index (BMI) was $26.71 \pm 3.89 \mathrm{~kg} / \mathrm{m}^{2}$. The frequency of smoking was $28 \%$ among the participants. A history of hypertension was reported in $38.7 \%$ of the patients, and a history of dyslipidemia was reported in 36\%. Besides, the history of myocardial infarction was reported in $4 \%$ of the patients, diabetes mellitus in $9.3 \%$ of the patients, and angioplasty in $6 \%$ of the patients. The CAC score only had significant relationships with hypertension and age (correlation coefficients $=0.51$ ). On the other hand, the AV-LV angle had no significant relationship with the patients' baseline characteristics. The statistical analysis of the AV-LV angle and CAC score showed no significant relationship between these parameters $(\mathrm{P}=0.756)$.

Conclusion: The AV-LV angle and the CAC score were not significantly associated. Further research is recommended to fill the knowledge gap regarding the cardiac anatomy features for cardiovascular risk assessment.
\end{abstract}

Keywords: Coronary Disease, Coronary Circulation, Cardiac Imaging Techniques, Computed Tomography

\section{Background}

Cardiovascular disease (CVD) is one of the leading causes of mortality in both developed and developing countries (1). Coronary artery disease (CAD) is described as a group of atherosclerotic diseases, involving inflammatory responses. It is associated with chest pain, heart attack, or sudden death $(2,3)$. According to previous reports, the prevalence of CAD is increasing around the world; therefore, identification of the associated risk factors and preventive care have become increasingly important $(4,5)$.

Changes in the blood flow of coronary arteries are one of the most important causes of CAD. Hypertension, diabetes, and hyperlipidemia are also among the common risk factors for CAD (6). Moreover, previous studies have proposed some new risk factors, such as aortic valve orientation. Today, orientation of the aortic valve is considered as an influential factor in the blood flow of coronary arteries (7-9). This change in the blood flow is caused by the conversion of the blood kinetic energy into hydraulic power, which leads to maximum flow and minimum pressure of the Venturi injector, affecting the blood flow into the distal part of the aorta $(10,11)$. 
On the other hand, studies on the fractional flow reserve (FFR) have revealed that changes in the blood flow of coronary arteries may be associated with vascular complications and the increased risk of coronary artery occlusion and cardiac ischemia (12). Moreover, some studies have shown that the presence of atherosclerotic plaques, vascular calcification, higher coronary artery calcium (CAC) scores, necrosis, and arterial remodeling can be associated with changes in the coronary artery blood flow and lead to acute coronary syndrome and sudden death (13).

According to previous studies, placement of an artificial valve in different orientations can change the blood pressure in the coronary arteries (14). In this regard, an ultrasonographic investigation of the coronary artery blood flow demonstrated a significant correlation between the aortic valve orientation and coronary blood flow (15). Another study, investigating the effect of the aortic valve angle on the left coronary artery blood flow, reported a significant relationship between the coronary artery pressure and the aortic valve angle, which could be attributed to the effect of the aortic valve angle on diastolic blood pressure (16). Evidence suggests that the aortic valve orientation can affect the blood flow of coronary arteries. It may be also accompanied by various vascular lesions that reduce the coronary artery blood flow and lead to heart attacks.

\section{Objectives}

The present study aimed to assess the relationship between the CAC score and the angle of the aortic valve with the left ventricular inflow long axis (AV-LV angle) as predictors of cardiovascular events.

\section{Patients and Methods}

\subsection{Ethical Considerations}

The Ethics Committee of Shahid Beheshti University of Medical Sciences (Tehran, Iran) approved this study (Ethics ID: IRIB.SBMU.MSP.REC.1397.326). Informed consent was obtained from all participants, and the collected data remained confidential.

\subsection{Study Design}

This single-center, prospective, cross-sectional study was conducted from July 2019 to January 2020. Seventyfour patients, admitted to Modarres Hospital (Tehran, Iran) with indications for coronary computed tomography angiography (CCTA), according to the American Heart Association (AHA) guidelines, were included in this study (17).
The exclusion criteria in the initial phase of the study were as follows: valve replacement; congenital heart disease; artificial heart valve; arrhythmia; creatinine level > $1.5 \mathrm{mg} / \mathrm{dL}$; allergic reactions to the contrast agent; pregnancy; left ventricular dilation (documented by echocardiography); body weight $\geq 100 \mathrm{~kg}$ (a limitation of CT machine); aortic regurgitation grade $3 / 4$; aortic stent; and history of coronary artery bypass graft (CABG). After collecting the required data, an experienced radiologist examined the radiographic images.

\subsection{Data Collection}

Questionnaires, including the patients' age, sex, height, weight, body mass index (BMI), smoking, history of high lipid profile, history of hypertension, history of myocardial infarction, history of angioplasty, family history of heart disease, and history of diabetes mellitus, were completed by conducting interviews with the patients.

\subsection{CT Imaging}

A 64-slice multi-detector CT scanner (Brilliance 64, Philips Medical System, Cleveland, OH, USA) was used for CT imaging (120 kVp, $380 \mathrm{~mA}$, collimation: $64 \times 0.625 \mathrm{~mm}$, increment: $0.33 \mathrm{~mm}$, pitch: 0.2 , and rotation time: $0.4 \mathrm{sec}$ ). CCTA was performed for all patients to measure the CAC score, as well as the aortic valve orientation. The CAC score was determined in the non-contrast phase. For this purpose, $320 \mathrm{mg} / \mathrm{mL}$ of Visipaque (containing $625 \mathrm{mg} / \mathrm{mL}$ of Iodixanol), was injected at a rate of $5.5 \mathrm{~mL} / \mathrm{sec}$. The CCTA images were reconstructed at $75 \%$ of the cardiac cycle.

Moreover, the Heartbeat-CS software (Philips Healthcare, the Netherlands) was used to evaluate non-contrast CCTA findings for determining the CAC score of each artery with a thickness of $2.5 \mathrm{~mm}$. In the Agatston method, the weighted sum of lesions with densities $>130$ Hounsfield units (HU) is measured, and the area of calcium is multiplied by a factor of maximum plaque attenuation: (1) factor 1, 130 - $199 \mathrm{HU}$; (2) factor 2, 200 - $299 \mathrm{HU}$; (3) factor 3, 300 - $399 \mathrm{HU}$; and (4) factor $4, \geq 400 \mathrm{HU}$ (18). In the horizontal long axis plane, a line was drawn from the center of the $\mathrm{mi}-$ tral valve to the apex of the LV. On the same plane, by moving through successive sections (from the section indicating both right and left coronary cusps), a line was drawn tangential to the inferior surface of the cusps. The angle between these two lines was measured as the AV-LV angle; Figure 1 illustrates these lines.

\subsection{Statistical Analysis}

The collected data are presented as mean \pm standard deviation (SD) or frequency (\%). Kolmogorov-Simonov test was used to evaluate the normal distribution of the AV-LV 

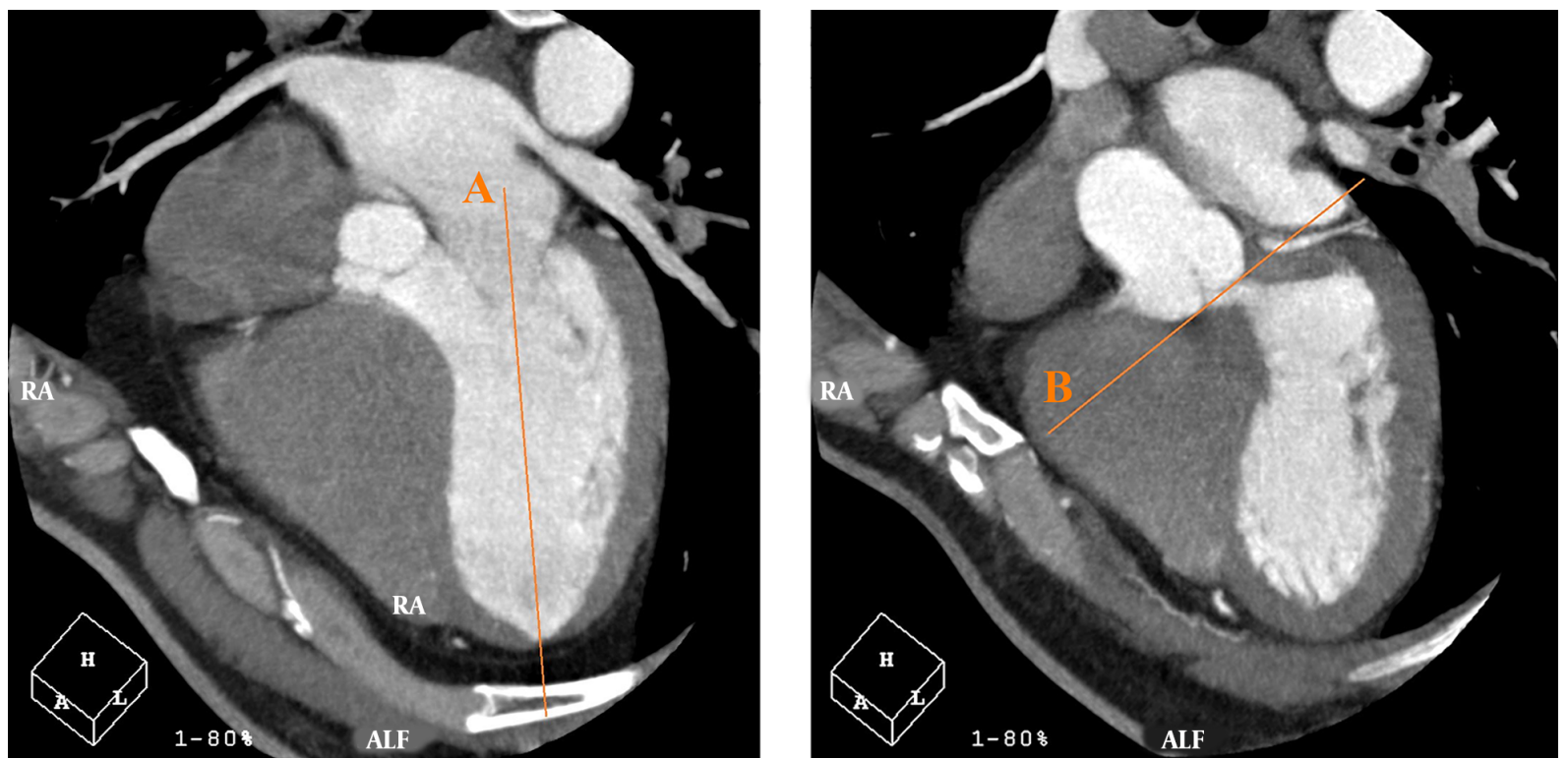

Figure 1. On the horizontal long axis plane, a line is drawn from the center of the mitral valve to the apex of the left ventricle (LV) (line A). On the same plane, by moving through successive sections, where both right and left coronary cusps can be seen, a line is drawn tangential to the inferior surface of the cusps (line B). The angle between these two lines is measured as the angle between the aortic valve and the left ventricular inflow long axis (AV-LV).

angles and CAC scores. Moreover, t-test and Mann-Whitney tests were used to compare the variables. Spearman's and Pearson's correlation coefficients were also measured to evaluate the correlations between non-normally and normally distributed data, respectively. A P-value less than 0.05 was considered significant. Data analysis was performed in SPSS version 24.0 (released in 2016, IBM SPSS Statistics for Windows, IBM Corp., Armonk, NY, USA).

\section{Results}

This study was performed on 74 participants (56\% male) with a mean age of $54.41 \pm 12.31$ years. The patient' mean height and weight were $167.07 \pm 15.11 \mathrm{~cm}$ and 75.97 $\pm 14.35 \mathrm{~kg}$, respectively. The mean AV-LV angle was 50.18 \pm 7.72 , and the mean CAC score was $75.97 \pm 14.35$. Table 1 shows the frequency of smoking (28\%), history of hypertension (38.7\%), history of dyslipidemia (36\%), history of myocardial infarction (4\%), and diabetes mellitus (9.3\%) among the participants. Overall, $45.3 \%$ of the patients had a family history of cardiac disease in their first-degree relatives. Also, 6 ( $8 \%$ ) patients had a history of angiography. Table 1 presents the relationship between the AV-LV angle and the patients' characteristics, including sex, age, BMI, smoking, hypertension, dyslipidemia, diabetes mellitus, myocardial infarction, and family history of CAD. The association between the CAC score and the mentioned characteristics is shown in Table 1.
No significant association was found between the AVLV angle and the baseline characteristics. Besides, the CAC score had no significant relationship with the patients' characteristics, except for hypertension and age $(\mathrm{P}=0.008$ and $\mathrm{P}=0.001$, respectively). Figure 2 presents the linear regression analysis of the AV-LV angle and CAC score, which did not indicate significant results $(\mathrm{P}=0.756)$.

\section{Discussion}

The present study examined the relationship between the CAC score and the AV-LV angle. This is the first study to evaluate the relationship between these two radiographic parameters. It was concluded that the CAC score did not have a significant relationship with the AV-LV angle. Although the CAC score has been described as one of the predictive factors for cardiovascular events, previous studies have not reported the AV-LV angle as a predictive factor (19).

CVD is one of the leading causes of mortality, accounting for about half of all mortality cases worldwide (20). According to the latest studies, at least $25 \%$ of non-fatal heart attacks or sudden cardiac deaths occur without any symptoms (21). Overall, diagnosis of asymptomatic and highrisk cases can reduce cardiovascular events in the future. Calculation of the risk score is a useful tool to classify patients, as it can predict 60 to $80 \%$ of cardiovascular events (18). The CAC score is one of the most useful tools for the early detection of CAD, calculated by CTA; it is almost as 


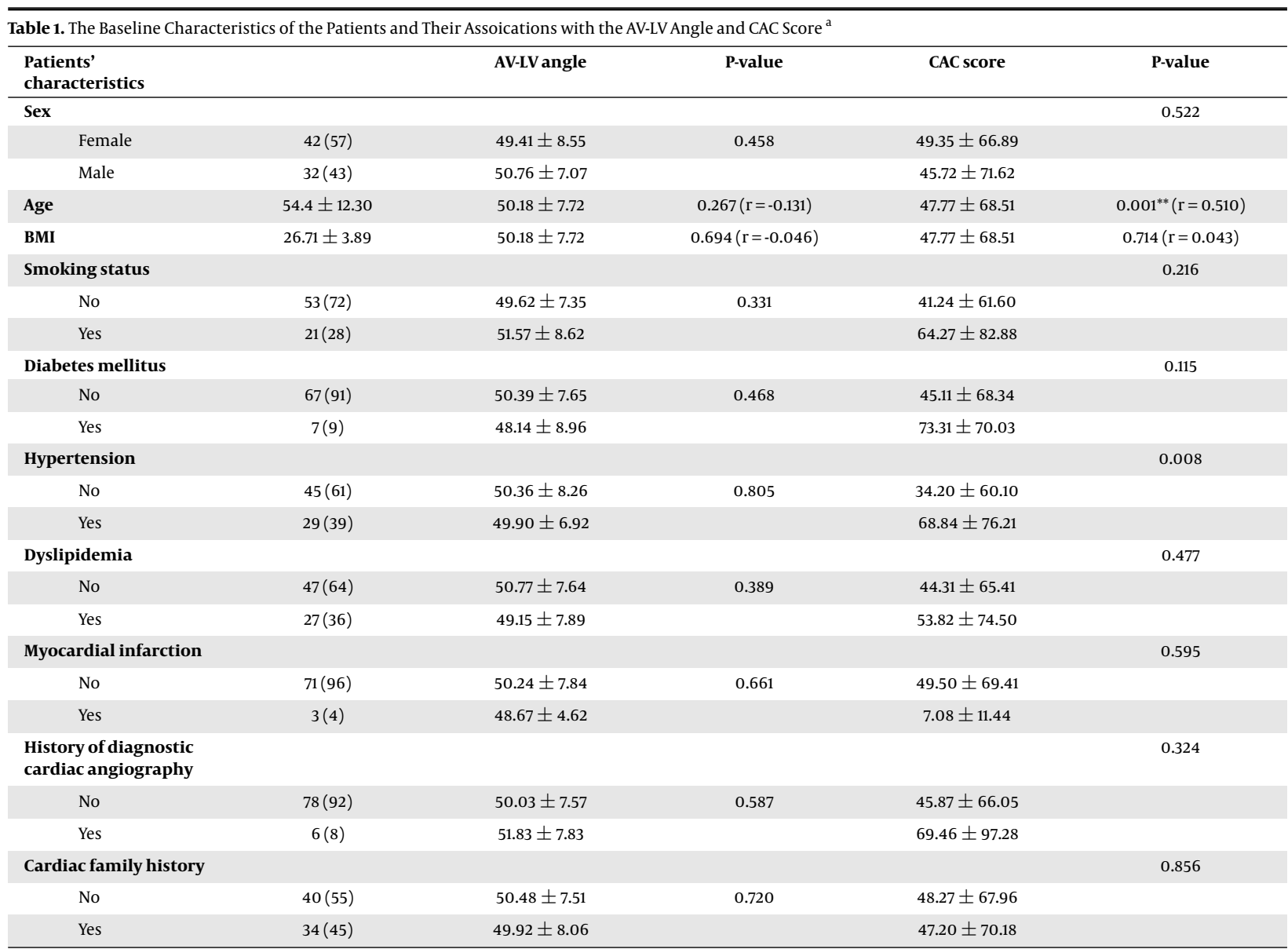

Abbreviations: AV-LV angle: angle between the aortic valve and the left ventricular inflow long axis; BMI, body mass index; CAC score, coronary artery calcium score.

${ }^{\mathrm{a}}$ Values are expressed as mean $\pm \mathrm{SD}$ or No. (\%).

valuable as risk scores, such as Framingham Risk Score. The CAC score was initially determined by electron beam computed tomography, although today, multi-detector CT imaging is used for its measurement (19).

Many studies have been conducted on the use of CAC scores for evaluating the cardiovascular risk factors. In a study by Greenland et al., the CAC scores were introduced as one of the available tools for the early detection of CAD, early and late prediction of cardiovascular events, early angiography interventions, and administration of aspirin (22). Moreover, a study by Elias-Smale found that the CAC scores were more accurate than the Framingham scores in the diagnosis of moderate-risk cases (23). However, the CAC score cannot rule out CAD, because in the early stages of CAD, this score may be as low as zero. Therefore, it is suggested to use this index along with other factors predicting coronary artery events (24).

The present study revealed that the CAC score had significant relationships with only hypertension and age, which is consistent with the findings of a study by LaMonte. On the other hand, the CAC score showed no significant relationship with BMI, sex, smoking, hyperlipidemia, myocardial infarction, family history of cardiac angioplasty, or diabetes mellitus, which is inconsistent with previous studies (25).

Another important factor in the early detection of CAD is the blood flow of coronary arteries, as reported in previous research. In a study by Dayanikli et al., the blood flow into the coronary arteries was evaluated among patients by positron emission tomography (PET). They concluded that a radiological examination of the coronary artery blood flow could be practical in predicting cardiovascular events. However, the coronary artery flow rate is different in patients with multiple risk factors, such as hyperlipidemia and older age; therefore, it can be used to predict cardiovascular events (26).

In this regard, an animal study concluded that the coronary blood flow was significantly influenced by the 


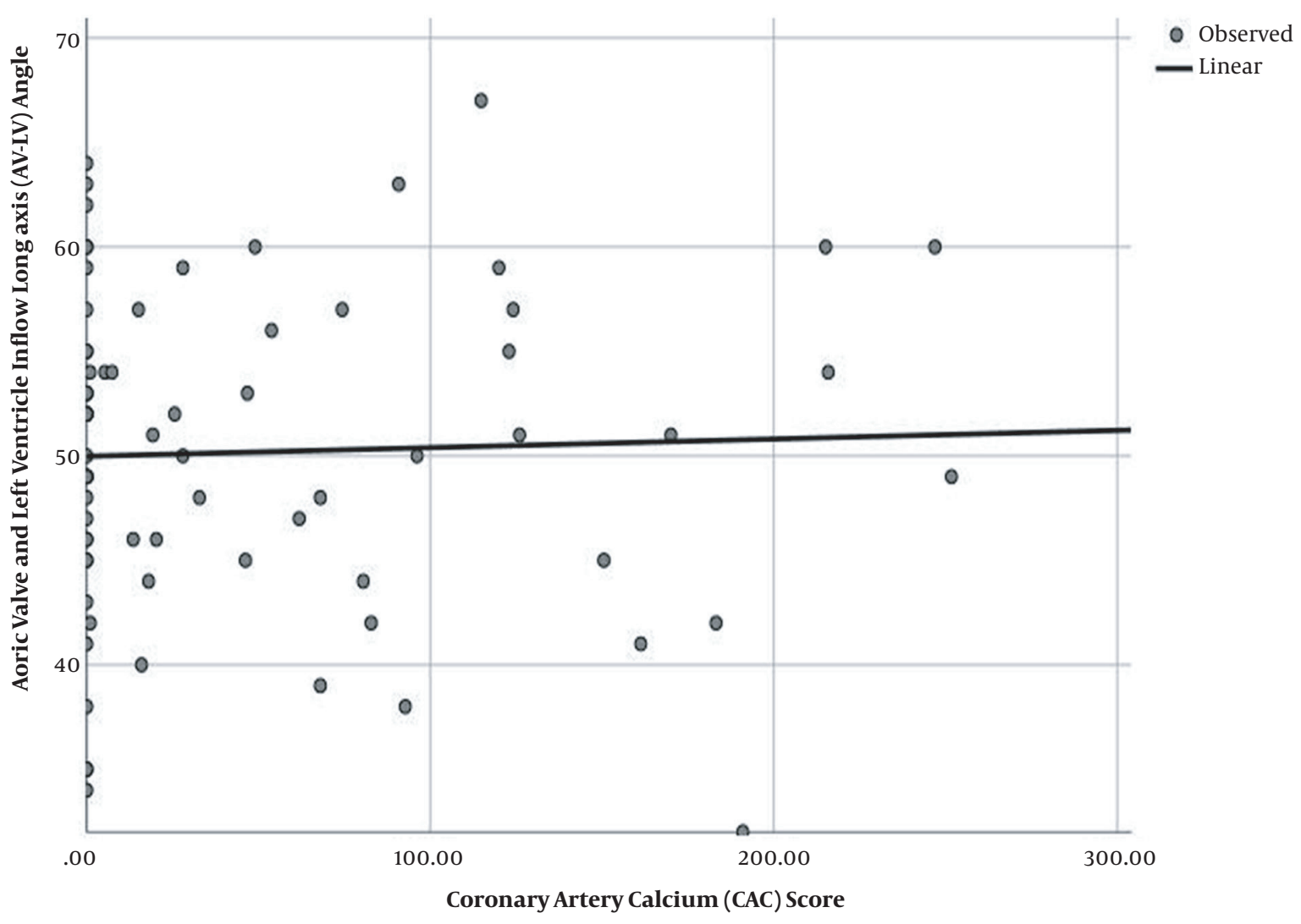

Figure 2. The linear regression analysis indicates no significant association between the coronary artery calcium (CAC) score and the angle of the aortic valve with the left ventricular inflow long axis $(\mathrm{AV}-\mathrm{LV})(\mathrm{P}=0.756, \mathrm{r}=0.024)$.

mechanical implantation of the aortic valve and the prosthetic mechanical valve orientation (9). Although the AVLV angle may play a role in coronary circulation, further investigations are needed. Also, calcification of the aortic valve, as one of the determinants of valve stenosis and one of the factors altering the coronary blood flow, can be useful in predicting CVD (27). Besides, the plaque volume and characteristics identified by CTA are important factors in the coronary artery blood flow, improving vascular lesion identification; therefore, early prevention of cardiac disease is possible (28).

In the present study, the AV-LV angle was not associated with the risk of cardiovascular events. In this regard, although an animal study on pigs showed that the implanted mechanical valve orientation could affect the valve performance, the AV-LV angle had no significant effects on the cardiac outcomes (9). The lack of a relationship between the AV-LV angle and the aortic artery flow contradicts our initial assumption. Nevertheless, van't Veer et al. concluded that valve orientation did not have a significant effect on the coronary artery blood flow (29). Another possible explanation for this finding is the aortic valve calcification, which has not been considered in the literature. Also, aortic valve stenosis seems to be related to calcification over time, with a greater effect on the incidence of CAD as compared to the AV-LV angle; however, further research is recommended.

It should be noted that in the present study, the angle between the aortic valve and the left ventricular axis was measured using a novel method, and further investigations are needed. Also, it was assumed that the AV-LV angle might be related to the coronary artery circulation, although further research is essential. Besides, some studies have examined other cardiac anatomy features in imaging to predict future cardiac events (i.e., aorta bifurcation angle, aortic valve orientation angle, angle between the left ventricle and aortic root, and thoracic aortic size) (30-32).

Additionally, this study aimed to investigate the correlation between the CAC score and the AV-LV angle. Since these parameters, especially the CAC sore, may play a role 
in predicting $\mathrm{CAD}$, we first investigated the correlation between these two parameters; however, no significant association was observed, which could be due to differences in their pathophysiology. Generally, the AV-LV angle is an anatomical feature, whereas the CAC score is representative of calcium plaques evolving over time.

There are some limitations in this study. The small sample size, lack of similar studies, absence of other radiologists for confirmation of radiological data, and use of operator-dependent methods are among these limitations. Future studies are recommended to fill the knowledge gap about cardiac anatomy features predicting cardiovascular risks. It is also recommended to examine the AV-LV angle and aortic valve calcification, along with the CAC score. Besides, inclusion of a separate group of patients with artificial aortic valves is suggested to examine the effect of AV-LV angle on the cardiac outcomes.

In conclusion, the AV-LV angle and the CAC score had no significant relationship. The AV-LV angle also had no significant association with other cardiovascular risk factors. However, a significant relationship was observed between the CAC score and age and also between the CAC score and hypertension.

\section{Footnotes}

Authors' Contribution: T.F.L., developed the original idea and the protocol with the help of A.E.; A.E., abstracted and collected data; F.Z.S. and S.A.A.S.N., wrote the manuscript; T.F.L. and A.E., re-evaluated the clinical data, revised the manuscript; T.F.L., examined the patients with the help of A.E.; S.A.A.S.N. and F.Z.S., prepared figures and tables. All authors discussed the results and commented on the manuscript.

Conflict of Interests: The authors disclose that they have no competing interests.

Ethical Approval: The Ethics Committee of Shahid Beheshti University of Medical Sciences (Tehran, Iran) approved this study (Ethics ID: IRIB.SBMU.MSP.REC.1397.326).

Funding/Support: The author(s) received no specific funding for this work.

Informed Consent: Informed consent was obtained from all participants, and the collected data remained confidential.

\section{References}

1. GBD Mortality Causes of Death Collaborators. Global, regional, and national age-sex specific all-cause and cause-specific mortality for 240 causes of death, 1990-2013: a systematic analysis for the Global Burden of Disease Study 2013. Lancet. 2015;385(9963):117-71. doi: 10.1016/S0140-6736(14)61682-2. [PubMed: 25530442]. [PubMed Central: PMC4340604].
2. Alvarez-Alvarez MM, Zanetti D, Carreras-Torres R, Moral P, Athanasiadis G. A survey of sub-Saharan gene flow into the Mediterranean at risk loci for coronary artery disease. Eur J Hum Genet. 2017;25(4):472-6. doi: 10.1038/ejhg.2016.200. [PubMed: 28098150]. [PubMed Central: PMC5386420].

3. Cassar A, Holmes DR, Rihal CS, Gersh BJ. Chronic coronary artery disease: diagnosis and management. Mayo Clin Proc. 2009;84(12):113046. doi: 10.4065/mcp.2009.0391. [PubMed: 19955250]. [PubMed Central: PMC2787400].

4. Krishnan MN, Zachariah G, Venugopal K, Mohanan PP, Harikrishnan $S$, Sanjay G, et al. Prevalence of coronary artery disease and its risk factors in Kerala, South India: A community-based cross-sectional study. BMC Cardiovasc Disord. 2016;16:12. doi:10.1186/s12872-016-0189-3. [PubMed: 26769341]. [PubMed Central: PMC4712491].

5. Lindahl B, Baron T, Erlinge D, Hadziosmanovic N, Nordenskjold A, Gard A, et al. Medical Therapy for Secondary Prevention and LongTerm Outcome in Patients With Myocardial Infarction With Nonobstructive Coronary Artery Disease. Circulation. 2017;135(16):1481-9. doi: 10.1161/CIRCULATIONAHA.116.026336. [PubMed: 28179398].

6. Malakar AK, Choudhury D, Halder B, Paul P, Uddin A, Chakraborty S. A review on coronary artery disease, its risk factors, and therapeutics. J Cell Physiol. 2019;234(10):16812-23. doi: 10.1002/jcp.28350. [PubMed: 30790284].

7. Yagi T, Yang W, Umezu M. Effect of Bileaflet Valve Orientation on the 3D Flow Dynamics in the Sinus of Valsalva.J Biomech Sci Eng. 2011;6(2):6478. doi: 10.1299/jbse.6.64.

8. Borazjani I, Sotiropoulos F. The effect of implantation orientation of a bileaflet mechanical heart valve on kinematics and hemodynamics in an anatomic aorta. J Biomech Eng. 2010;132(11):111005. doi: 10.1115/1.4002491. [PubMed: 21034146]. [PubMed Central: PMC3139272].

9. Kleine P, Scherer M, Abdel-Rahman U, Klesius AA, Ackermann H, Moritz A. Effect of mechanical aortic valve orientation on coronary artery flow: comparison of tilting disc versus bileaflet prostheses in pigs. J Thorac Cardiovasc Surg. 2002;124(5):925-32. doi: 10.1067/mtc.2002.126046. [PubMed:12407375].

10. Fiore GB, Grigioni M, Daniele C, D’Avenio G, Barbaro V, Fumero R. Hydraulic functional characterisation of aortic mechanical heart valve prostheses through lumped-parameter modelling. J Biomech. 2002;35(10):1427-32. doi: 10.1016/s0021-9290(02)00177-x. [PubMed: 12231289].

11. Tweddell JS, Pelech AN, Frommelt PC. Ventricular septal defect and aortic valve regurgitation: pathophysiology and indications for surgery. Semin Thorac Cardiovasc Surg Pediatr Card Surg Annu. 2006:14752. doi: 10.1053/j.pcsu.2006.02.020. [PubMed:16638560].

12. Virmani R, Kolodgie FD, Burke AP, Farb A, Schwartz SM. Lessons from sudden coronary death: A comprehensive morphological classification scheme for atherosclerotic lesions. Arterioscler Thromb Vasc Biol. 2000;20(5):1262-75. doi: 10.1161/01.atv.20.5.1262. [PubMed: 10807742].

13. Virmani R, Burke AP, Farb A, Kolodgie FD. Pathology of the vulnerable plaque. J Am Coll Cardiol. 2006;47(8 Suppl):C13-8. doi: 10.1016/j.jacc.2005.10.065. [PubMed: 16631505].

14. Akutsu T, Saito J, Imai R, Suzuki T, Cao XD. Dynamic particle image velocimetry study of the aortic flow field of contemporary mechanical bileaflet prostheses. J Artif Organs. 2008;11(2):75-90. doi: 10.1007/s10047-008-0410-y. [PubMed: 18604612].

15. Akutsu T, Matsumoto A, Takahashi K. In Vitro Study of the Correlation between the Aortic Flow Field Affected by the Bileaflet Mechanical Valves and Coronary Circulation.In: Jobbágy Á, editor. 5th European Conference of the International Federation for Medical and Biological Engineering. Budapest, Hungary. 2011. p. 769-72.

16. Tonino PA, De Bruyne B, Pijls NH, Siebert U, Ikeno F, van' t Veer M, et al. Fractional flow reserve versus angiography for guiding percutaneous coronary intervention. $N$ Engl J Med. 2009;360(3):213-24. doi: 10.1056/NEJMoa0807611. [PubMed: 19144937]. 
17. Bittencourt MS, Hulten EA, Veeranna V, Blankstein R. Coronary Computed Tomography Angiography in the Evaluation of Chest Pain of Suspected Cardiac Origin. Circulation. 2016;133(20):1963-8. doi: 10.1161/CIRCULATIONAHA.116.017593. [PubMed: 27185023].

18. Neves PO, Andrade J, Moncao H. Coronary artery calcium score: current status. Radiol Bras. 2017;50(3):182-9. doi: 10.1590/01003984.2015.0235. [PubMed: 28670030]. [PubMed Central: PMC5487233]

19. Budoff MJ, Achenbach S, Blumenthal RS, Carr JJ, Goldin JG, Greenland $\mathrm{P}$, et al. Assessment of coronary artery disease by cardiac computed tomography: A scientific statement from the American Heart Association Committee on Cardiovascular Imaging and Intervention, Council on Cardiovascular Radiology and Intervention, and Committee on Cardiac Imaging, Council on Clinical Cardiology. Circulation. 2006;114(16):1761-91. doi: 10.1161/CIRCULATIONAHA.106.178458. [PubMed: 17015792]

20. Nasir K, Clouse M. Role of nonenhanced multidetector CT coronary artery calcium testing in asymptomatic and symptomatic individuals. Radiology. 2012;264(3):637-49. doi: 10.1148/radiol.12110810. [PubMed: 22919038].

21. Greenland P, Smith SJ, Grundy SM. Improving coronary heart disease risk assessment in asymptomatic people: role of traditional risk factors and noninvasive cardiovascular tests. Circulation. 2001;104(15):1863-7. doi: 10.1161/hc4201.097189. [PubMed: 11591627].

22. Greenland P, Blaha MJ, Budoff MJ, Erbel R, Watson KE. Coronary Calcium Score and Cardiovascular Risk. J Am Coll Cardiol. 2018;72(4):43447. doi: 10.1016/j.jacc.2018.05.027. [PubMed:30025580]. [PubMed Central: PMC6056023].

23. Elias-Smale SE, Proenca RV, Koller MT, Kavousi M, van Rooij FJ, Hunink MG, et al. Coronary calcium score improves classification of coronary heart disease risk in the elderly: The Rotterdam study. J Am Coll Cardiol. 2010;56(17):1407-14. doi: 10.1016/j.jacc.2010.06.029. [PubMed: 20946998].

24. Senoner T, Plank F, Beyer C, Langer C, Birkl K, Steinkohl F, et al. Does coronary calcium score zero reliably rule out coronary artery disease in low-to-intermediate risk patients? A coronary CTA study. J Cardiovasc Comput Tomogr. 2020;14(2):155-61. doi: 10.1016/j.jcct.2019.09.009. [PubMed: 31570324]
25. LaMonte MJ, FitzGerald SJ, Church TS, Barlow CE, Radford NB, Levine $\mathrm{BD}$, et al. Coronary artery calcium score and coronary heart disease events in a large cohort of asymptomatic men and women. Am J Epidemiol. 2005;162(5):421-9. doi: 10.1093/aje/kwi228. [PubMed: 16076829].

26. Dayanikli F, Grambow D, Muzik O, Mosca L, Rubenfire M, Schwaiger M. Early detection of abnormal coronary flow reserve in asymptomatic men at high risk for coronary artery disease using positron emission tomography. Circulation. 1994;90(2):808-17. doi: 10.1161/01.cir.90.2.808. [PubMed: 8044952].

27. Wu VC, Takeuchi M, Nagata Y, Izumo M, Akashi YJ, Lin FC, et al. Prognostic value of area of calcified aortic valve by 2-dimensional echocardiography in asymptomatic severe aortic stenosis patients with preserved left ventricular ejection fraction. Medicine (Baltimore). 2018;97(12). e0246. doi: 10.1097/MD.0000000000010246. [PubMed: 29561453]. [PubMed Central: PMC5895302].

28. Park HB, Heo R, O. Hartaigh B, Cho I, Gransar H, Nakazato R, et al. Atherosclerotic plaque characteristics by $\mathrm{CT}$ angiography identify coronary lesions that cause ischemia: a direct comparison to fractional flow reserve. JACC Cardiovasc Imaging. 2015;8(1):1-10. doi: 10.1016/j.jcmg.2014.11.002. [PubMed: 25592691]. [PubMed Central: PMC4297319].

29. van't Veer M, van Straten B, vande Vosse F, Pijls N. Influence of orientation of bi-leaflet valve prostheses on coronary perfusion pressure in humans. Interact Cardiovasc Thorac Surg. 2007;6(5):588-92. doi: 10.1510/icvts.2007.156877. [PubMed: 17670729].

30. Shakeri AB, Tubbs RS, Shoja MM, Nosratinia H, Oakes WJ. Aortic bifurcation angle as an independent risk factor for aortoiliac occlusive disease. Folia Morphol (Warsz). 2007;66(3):181-4. [PubMed: 17985315].

31. Roule V, Placente A, Sabatier R, Bignon M, Saplacan V, Ivascau C, et al. Angles between the aortic root and the left ventricle assessed by MDCT are associated with the risk of aortic regurgitation after transcatheter aortic valve replacement. Heart Vessels. 2018;33(1):58-65. doi: 10.1007/s00380-017-1032-1. [PubMed: 28799113].

32. Hardikar AA, Iannaccone A, Marwick TH. Abstract 18662: Aortic Valve Orientation Angle is Associated With Aortic Morphology and Dissection Risk. Circulation. 2014;130(Suppl 2). A18662. doi: 10.1161/circ.130.suppl_2.18662. 